\title{
A thrombotic complication of COVID-19: cerebral ischaemia in the intensive care unit
}

Ayça Sultan Şahin ${ }^{1}$, Kader Irak², Ebru Kaya ${ }^{1}$, Sureyya Ozkan ${ }^{1}$, Fikret Baskan ${ }^{3}$

\author{
${ }^{1}$ Department of Anesthesiology and Reanimation, Kanuni Sultan Suleyman Education \\ and Training Hospital, İstanbul, Turkey \\ ${ }^{2}$ Department of Intensive Medicine-Gastroenterology, Kanuni Sultan Suleyman \\ Education and Training Hospital, İstanbul, Turkey \\ ${ }^{3}$ Department of Neurosurgery, Kanuni Sultan Suleyman Education and Training \\ Hospital, İstanbul, Turkey
}

Submitted: 20 July 2020

Accepted: 25 July 2020

Arch Med Sci Atheroscler Dis 2020; 5: e224-e225

DOI: https://doi.org/10.5114/amsad.2020.98920

Copyright @ 2020 Termedia \& Banach

COVID-19 may predispose to thromboembolic disease due to severe inflammation, hypoxia, and immobilisation [1]. The severe hypercoagulable state of COVID-19 patients includes systemic micro- and macrovascular thrombosis. Anticoagulant therapy, mainly with low-molecular-weight heparin, appears to be associated with better prognosis in severe COVID-19 patients [2].

A 45-year-old non-smoking male patient with no chronic disease and normal laboratory blood levels presented with fever, cough, and dyspnoea. Echocardiography did not show atrial fibrillation or atherosclerotic plaque. COVID-19 was diagnosed based on RT-PCR testing. There were no clinical or laboratory signs of a thromboembolic state, and the patient had no history of disseminated intravascular coagulation, venous thromboembolism, or patent foramen ovale shunt. After 4 days, the patient lost consciousness and had a seizure. The patient's illness subsequently progressed to respiratory failure, warranting the initiation of non-invasive mechanical ventilation in the intensive care unit (ICU). Figure 1 depicts the diffusion brain magnetic resonance imaging (MRI) (T2-sequence) with no contrast and shows hyperintense areas in the cortical and subcortical areas at the left temporofrontoparietal level and at the left lentiform nucleus level. Pulmonary angiography (Figure 2) findings were compatible with thromboembolism in the right and left lung bases. In the ICU, the patient received antithrombotic therapy: enoxaparin sodium (6000 anti-Xa/0.6 ml, twice a day) and acetylsalicylic acid $100 \mathrm{mg}$. Levetiracetam $250 \mathrm{mg}$ twice a day was started for seizures. On April 3, 2020, the patient was transferred to the neurological ward. He had right hemiplegia and aphasia.

Available evidence shows that markedly elevated D-dimer levels are associated with high mortality in COVID-19 patients [3]. Multi-organ failure is more likely in patients with sepsis if they develop coagulopathy, and inhibiting thrombin generation may have benefits in reducing mortality [4]. If there are no contraindications for patients requiring hospital admission for COVID-19 infection, prophylactic doses of low-molecular-weight heparin should be administered. The prophylactic enoxaparin dose recommended by the International Society of Thrombosis and Haemostasis on March 25, 2020 is 4000 U [3]. However, we suggest that this prophylactic dose is not sufficient to protect against thromboembo-

\author{
Corresponding author: \\ Assoc. Prof. Dr. Ayça Sultan \\ Şahin \\ Department \\ of Anesthesiology \\ and Reanimation \\ Kanuni Sultan Suleyman \\ Education and \\ Training Hospital \\ Istanbul, Turkey \\ Phone: +90505 3980419 \\ E-mail: aycasultan@gmail. \\ com
}




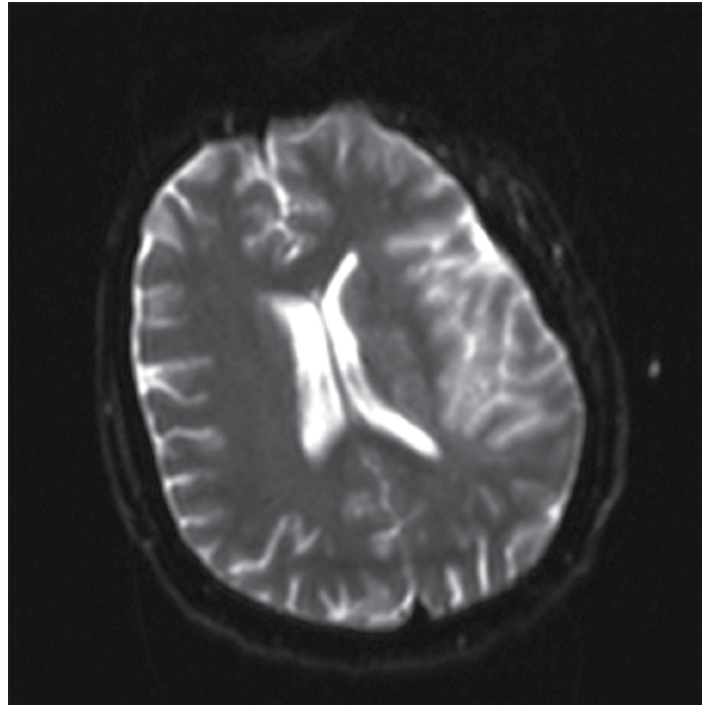

Figure 1. Diffuse MRI-T2 sequence

lism. In many ICUs, higher doses have been used with the same rationale, confirmed by the data from Klok et al. from 184 patients, in whom most events (81\%) were pulmonary embolism [5].

Antiphospholipid antibodies are common in the general population, especially during infection [6]. COVID-19 may induce a hypercoagulable state with elevated fibrinogen and prolonged PT and aPTT. The mechanisms underlying coagulopathy are not clear [7]. Zhang et al. reported that SARS-CoV-2 infection is associated with coagulopathy, thrombocytopaenia, and the presence of anticardiolipin IgA, anti- 2-glycoprotein I, IgA, and IgG antibodies, all of which are responsible for cerebral infarcts [8]. Lodigiani et al. analysed the rate, timing, and characteristics of venous/arterial thromboembolic complications among COVID-19 patients. Their results revealed that thromboembolic complications might represent an integral part of the COVID-19 disease and may already be present at the time of initial hospital admission [9]. We suggest that hypercoagulability caused by COVID-19 may cause ischaemia not only in the lungs but also in vital organs such as the brain.

\section{Conflict of interest}

The authors declare no conflict of interest.

\section{References}

1. Zhou F, Yu T, Du R, et al. Clinical course and risk factors for mortality of adult inpatients with COVID-19 in Wuhan, China: a retrospective cohort study. Lancet 2020; 395: 1054-62.

2. Tang N, Bai H, Chen X, Gong J, Li D, Sun Z. Anticoagulant treatment is associated with decreased mortality in severe coronavirus disease 2019 patients with coagulopathy. J Thromb Haemost 2020; 18: 1094-9.

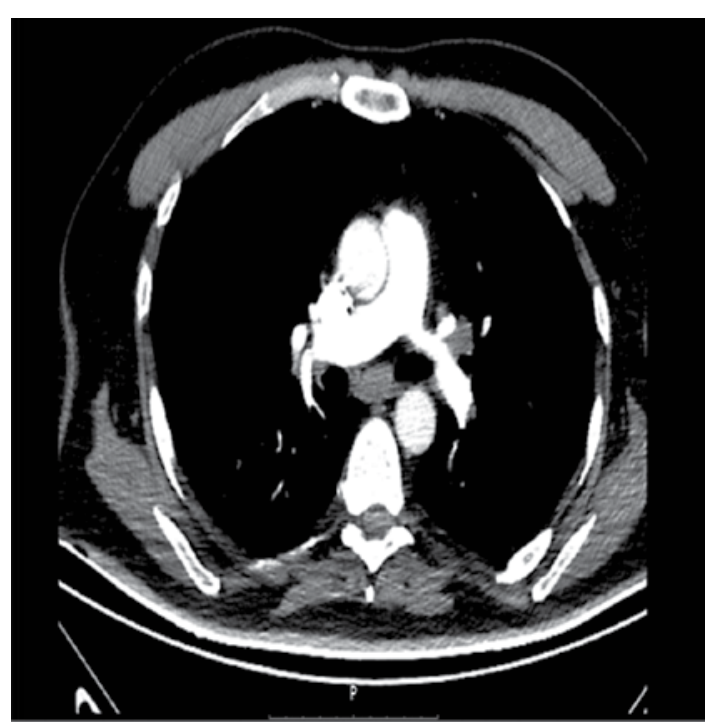

Figure 2. CT angiography scan

3. Thachil J, Tang N, Gando S, et al. ISTH interim guidance on recognition and management of coagulopathy in COVID-19. J Thromb Haemost 2020; 18: 1023-6.

4. Shankar-Hari M, Phillips GS, Levy ML, et al.; Sepsis Definitions Task Force. Developing a new definition and assessing new clinical criteria for septic shock: for the Third International Consensus Definitions for Sepsis and Septic Shock (Sepsis-3). JAMA 2016; 315: 775-87.

5. Klok FA, Kruip MJHA, van der Meer NJM, et al. Incidence of thrombotic complications in critically ill ICU patients with COVID-19. Thromb Res 2020; 191: 145-7.

6. Connell NT, Battinelli EM, Connors JM. Coagulopathy of COVID-19 and antiphospholipid antibodies. J Thromb Haemost 2020 May 7. doi: 10.1111/jth.14893.

7. Tang N, Li D, Wang X, Sun Z. Abnormal coagulation parameters are associated with poor prognosis in patients with novel coronavirus pneumonia. J Thromb Haemost 2020; 18: 844-7.

8. Zhang Y, Xiao M, Zhang S, et al. Coagulopathy and antiphospholipid antibodies in patients with COVID-19. N Engl J Med 2020; 382: e38.

9. Lodigiani C, Iapichino G, Carenzo L, et al. Venous and arterial thromboembolic complications in COVID-19 patients admitted to an academic hospital in Milan, Italy. Thromb Res 2020; 191: 9-14. 